\title{
Artificial Intelligence in Ophthalmology
}

\author{
Tayyaba Gul Malik ${ }^{1}$ \\ ${ }^{1}$ Department of Ophthalmology, Rashid Latif Medical College, Lahore
}

Many of us have read or heard the famous story of novel "Frankenstein" written in 1818 by Mary Shelley. ${ }^{1}$ The story was about an artificial human or a human-like creature made by a young scientist. Although this creature had nothing similar to today's artificial intelligence (AI), but that can be referred to as the first $\mathrm{AI}$ in fiction stories. This was the time when the first industrial revolution (1760-1840) was taking place with new materials made of iron and steel. Later, AI took the form of machines and robots. Starting from movie by the name of "Metropolis" in 1927 to the latest movie released in 2020 by the name of "super intelligence" there is a long journey of fiction stories on artificial intelligence. However, it is no longer a fiction in the twenty first century.

In the real world of science, the word 'artificial intelligence' was introduced 70 years back in 1950s. It was a software, which had the capability of learning from experience and problem solving by processing and recognizing different patterns of huge amounts of data. $^{2}$

Before the role of AI in ophthalmology could be talked about, it is pertinent to know the different types of AI. The simplest example of AI is the google browser system. Google search engine gives recommendations once you type a word in the search bar. It depends on a set of input data on the basis of which, outcome is predicted. These predictions are based on the information that it collects from our search history, location and age. Machine learning (ML) and Deep learning (DL) are other frequently

How to Cite this Article: Malik TG. Artificial Intelligence in Ophthalmology. Pak J Ophthalmol. 2021, 37 (1): 1-3.

Doi: https://doi.org/10.36351/pjo.v37i1.1170

Correspondence: Tayyaba Gul Malik

Department of Ophthalmology, Rashid Latif Medical College, Lahore

Email:tayyabam@yahoo.com used terms in AI. Machine learning refers to a process by which we feed data into a machine and the machine devices a strategy on its own to perform a certain task. In health care system, we can give example of $2 \mathrm{D}$ or 3D images of patients (retinal cameras, X-rays, CT scans etc.) which are called INPUTS or training datasets. On the basis of these inputs, machine trains itself to make a diagnosis in the form of OUTPUTS. In comparison to ML, DL works just like human brain by using complex neural network $(\mathrm{CNN})$ after receiving multiple input data. Examples include recognition of objects in images, language translation in real-time, manipulation of electronic devices by speech and so on and so forth. A larger set of data is required in this case. In simplest terms, the difference between ML and DL is that ML consists of input and output while DL comprises of input, hidden layer/layers (which carry out various computations) and then output. ${ }^{3}$

Different models of AI are identified. The fullyautomated and semi-automated models are the two frequently used ones. In fully automated model, the machine identifies a disease and suggests the referral or further monitoring by its own. However, in semiautomated models, a human grader is needed to suggest intervention, referral or monitoring.

Use of AI in Ophthalmology involves different algorithms and many of which are in the experimental phase to help in screening, diagnosing and monitoring major eye diseases. The whole process of identification of disease can be categorized into four steps. Collection of large amount of images and input data is the kick-off step. Second step involves labelling of ocular lesions by the ophthalmologists. In the third step, the computer program extracts salient features of the disease from the images or data provided. Fourth and the final step is to give an output. By 2018, PubMed library showed 243 articles of AI application in diagnosing ocular diseases. ${ }^{4}$

Although literature describes various fields of ophthalmology in which AI is being tried, system for 
Diabetic Retinopathy (DR) was the first to be approved by FDA in April 2018 (IDx-DR system). This device has a sensitivity and specificity of $87.3 \%$ and $89.5 \%$, respectively. Use of this device can help in increasing the number of patients who can get screened at primary care. Although some data shows that AI performs better than ophthalmologists in early detection of DR but more clinical data is still needed to prove that. ${ }^{5}$

Other areas include age-related macular degeneration, retinopathy of prematurity, glaucoma, keratoconus, retinal detachment, retinal vascular occlusions, cataract, squint, refractive errors, and ocular oncology. ${ }^{6,7}$ In early 2020, I-ROP received Breakthrough Device status by FDA. It has been claimed that I-ROP is capable of identifying plus disease better than the expert ophthalmologist.

For diagnosis of glaucoma, AI DL models use fundus images with optic disc changes, OCT scans, result of visual fields, intraocular pressure and corneal thickness. ${ }^{8}$ Detection and grading of cataract has also been made possible by using ML programs. Res Net system is a unique model capable of identifying referable cataracts. There is another program called CC-Cruiser, which is based on Slit lamp photographs to identify the density and degree of congenital cataract. Role of $\mathrm{AI}$ in calculating $\mathrm{IOL}$ is also commendable. The common example is Hill-RBF formula, which uses axial length, central corneal thickness, anterior chamber depth, lens thickness, corneal diameter, and keratometry measurements to calculate the lens power with great accuracy. ${ }^{9}$

AI has also made striking progress in ocular oncology. It has been employed to predict the results of periocular reconstruction in cases of BCC, which helps in decision making after removal of tumor. Some AI models can help to prognosticate the outcomes of choroidal melanoma using demographic data and oncologic history. Another development in ocular oncology is the non-invasive detection of ocular surface squamous neoplasia by making use of multispectral auto-fluorescence imaging. ${ }^{10}$

A deep learning algorithm for the assessment of strabismus using photographic images of patients is also being tried. Similarly, detection of refractive errors can also be possible with AI techniques one of which is based on Brückner pupil red reflex imaging. ${ }^{11.12}$ Work on retinal vein occlusion is also underway and the initial results are very encouraging.
AI has revolutionized the medical field during the last decade but its accuracy depends on the input data. 'Garbage in Garbage out' phenomenon holds true for it. That is not all, development of an algorithm requires huge costs and experience. Thus, right now such investments can only be made for very common diseases with high morbidity and mortality. Similarly, as the AI is based on the input data and there are variations in different disease presentations in different populations, the data cannot be generalized. For accurate results whole set of new data from different population has to be incorporated into the machine. Similarly, any variation of a disease, which is not included in the input data will be missed by the machine. Variations in the geographical areas with unequal facilities of healthcare is another hurdle in implementation of $\mathrm{AI}^{13}$ Another concern is that an ophthalmologist sees a patient for all kinds of ocular diseases. However, when it was tried to incorporate more diseases into a single machine, the accuracy of detection was decreased. A particular algorithm is designed to detect only one disease at a time. For example, a model which is designed for ROP will fail to detect glaucoma as a disease or abnormality. In future these problems will be solved.

Although application of AI has facilitated tele health programs and has overcome the issue of shortage of specialists, there are liability issues as well. Most important is the case if an error is made, would the clinician be liable or the AI technology programmer. Such questions need to be answered and laws are yet to be made before widespread implementation of AI. There are other issues related with the consent, privacy and security of the patients as well.

Advent of AI has revolutionized the field of medicine and further work is being done for refining the results and decreasing the dependency on humans, but somehow some kind of human intervention will always be needed.

\section{Conflict of Interest}

Authors declared no conflict of interest.

\section{REFERENCES}

1. Frankenstein. Available at: https://en.wikipedia.org/wiki/Frankenstein. Accessed on 19. November 2020. 
2. Kapoor R, Walters SP, Al-Aswad LA. The current state of artificial intelligence in ophthalmology. Surv Ophthalmol. 2019; 64 (2): 233-240.

Doi:10.1016/j.survophthal.2018.09.002

3. Ting DSW, Peng L, Varadarajan AV, Keane PA, Burlina PM, Chiang MF, et al. Deep learning in ophthalmology: The technical and clinical considerations. Prog Retin Eye Res. 2019.

Doi: 10.1016/j.preteyeres.2019.04.003

4. Lu W, Tong Y, Yu Y, Xing Y, Chen C, Shen Y. Applications of Artificial Intelligence in Ophthalmology: General Overview. J Ophthalmol. 2018; 5278196: 15. https://doi.org/10.1155/2018/5278196

5. Abràmoff MD, Lavin PT, Birch M, Shah N, Folk JC. Pivotal trial of an autonomous AI-based diagnostic system for detection of diabetic retinopathy in primary care offices. NPJ Digit Med. 2018; 1: 39.

Doi: 10.1038/s41746-018-0040-6

6. Hwang DK, Hsu CC, Chang KJ, Chao D, Sun CH, Jheng YC, et al. Artificial intelligence-based decisionmaking for age-related macular degeneration. Theranostics, 2019; 9: 232-245.

7. Brown JM, Campbell JP, Beers A, Chang K, Ostmo S, Chan RVP, et al. Automated Diagnosis of Plus Disease in Retinopathy of Prematurity Using Deep Convolutional Neural Networks. JAMA Ophthalmol. 2018; 136 (7): 803-810.

Doi: 10.1001/jamaophthalmol.2018.1934
8. Schmidt-Erfurth U, Sadeghipour A, Gerendas BS, Waldstein SM, Bogunović $\mathbf{H}$. Artificial intelligence in retina. Prog Retin Eye Res. 2018; 67: 1-29.

9. Liu X, Jiang J, Zhang $\mathbf{K}$, et al. Localization and diagnosis framework for pediatric cataracts based on slit-lamp images using deep features of a convolutional neural network. PLoS One, 2017; 12 (3): e0168606. Doi: 10.1371/journal.pone.0168606

10. Habibalahi A, Bala C, Allende A, Anwer AG, Goldys EM. Novel automated non invasive detection of ocular surface squamous neoplasia using multispectral autofluorescence imaging. Ocul Surf. 2019; 17 (3): 540-550.

Doi: $10.1016 /$ j.jtos.2019.03.003

11. Chen Z, Fu H, Lo W-L, Chi Z. Strabismus Recognition Using Eye-Tracking Data and Convolutional Neural Networks. J Healthc Eng. 2018; 2018: 7692198. Doi: $10.1155 / 2018 / 7692198$

12. Reid JE, Eaton E. Artificial intelligence for pediatric ophthalmology. Curr Opin Ophthalmol. 2019; 30 (5): 337-346. Doi: 10.1097/ICU.0000000000000593

13. Du XL, Li WB, Hu BJ. Application of artificial intelligence in ophthalmology. Int J Ophthalmol. 2018; 11 (9): 1555-1561. Doi: 10.18240/ijo.2018.09.21. 